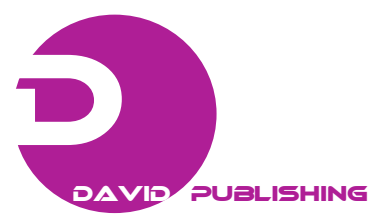

\title{
Overview of Concentrated Solar Power
}

\author{
Chukwubuikem Chukwuka and Komla Agbenyo Folly \\ Faculty of Engineering and the Built Environment, University of Cape Town, Rondebosch 7701, South Africa
}

Received: May 11, 2013 / Accepted: June 06, 2013 / Published: December 31, 2013.

\begin{abstract}
CSP (concentrated solar power) has been viewed as the technology that if properly developed could lead to a large scale conversion of solar energy into electricity. CSP is a type of solar energy converter that is classified as thermal converter because the output power produced is a function of the operating temperature. The main components of a CSP plant are the solar field which is made up of the heliostat arrays, the receiver tower, the heat transfer fluid, the molten salt thermal energy storage tanks and the power conversion unit, which is made up of the turbine and the generator. The main advantage of CSP is that of a cheap thermal storage (i.e., molten salt storage) which makes it possible to dispatch power at a cost comparable to the grid electricity. Simulations run with the SAM (systems advisory model) developed by NREL (National Renewable Energy Laboratory) showed that CSP is capable of delivering electricity at the cost of $17 \mathrm{UScents}$ per $\mathrm{kWh}$ for the 30 -year life of the plant. The main disadvantage of CSP however, is that of low efficiency $(8 \%-16 \%)$. There are ongoing research works to improve the efficiency of the CSP. One way to improve the efficiency is to increase the operating temperature of the system. In this paper, the authors discussed different modules of the CSP plant and suggested ways to improve on the conversion efficiencies of individual modules. Finally, an overall systems performance simulation is carried using SAM and the simulation results show that electricity can be produced using CSP at the cost of R1.05 per kWh.
\end{abstract}

Key words: Concentrated solar power, heliostat, molten salt energy storage, SAM (systems advisory model), LCOE (levelised cost of electricity), blackbody receiver/emitter.

\section{Introduction}

Concentrated solar power technology is a ripe technology capable of producing clean, renewable and grid-scale electricity especially, in the African region which has days and weeks of direct sunshine and DNI (direct normal irradiance) of $2,816 \mathrm{kWh} / \mathrm{m}^{2}$. Three different types of CSP (concentrated solar power) technologies have been developed: the parabolic trough, the power tower and the parabolic dish. The parabolic trough is the most mature and commercially available design [1]. The parabolic trough is the cheapest but also the least efficient (typically about $11 \%$ ). The power tower is the least developed type but it has an advantage of reaching higher temperatures which could mean higher efficiency considering the fact that in thermal converters the efficiency is proportional to the operating temperature. Several

Corresponding author: Komla Agbenyo Folly, associate professor, research fields: power system stability and control, renewable energy. E-mail: Komla.Folly@uct.ac.za.
CSPs have been already installed and running around the world today and these include the Solar 1 in Mojave Desert in California which has been upgraded to Solar 2 and the Solar Tres in Spain. These are experimental pilot projects. South Africa is planning to build a 100 MW CSP in Upington in Northern Cape Province. In this paper, the authors discussed different modules of the CSP plant and suggested ways to improve on the conversion efficiencies of individual modules. Finally, an overall systems performance simulation is carried using SAM (Systems Advisory Model). The paper is organized as follows: Section 2 deals with a general overview of the concentrating solar power while Section 3 describes the working principles of power tower CSP. Section 4 discusses briefly the simulation results of molten salt power tower CSP, and conclusion is given in Section 5 .

\section{Overview of Concentrated Solar Power}

The technology of converting solar energy to 
electricity via thermal energy is referred to as the concentrated solar power. Globally, there are up to 600 MW of installed CSP running by 2009 and $15 \mathrm{GW}$ being planned and under construction [1]. The first oil crisis in the early 1970s marked the beginning of modern development of CSP plants worldwide. The first nine CSP test projects where developed in Mojave Desert of California and they were named SEGS I-IX (Solar Energy Generating Systems). SEGS I-II with the capacity of $44 \mathrm{MW}$ are located in Daggett, California. From 1986 to 1988, SEGS III-VII were built in Krammer Junction, California. They are made up of six CSPs with a total capacity of 150 MW. Between 1989 and 1990, SEGS VIII-IX were built in Harper Lake with the total capacity of $160 \mathrm{MW}$. All of these plants operate at an annual efficiency of less than $15 \%$. Other CSP plants are in Spain, Italy, China and India. Here in South Africa, Eskom (The South African Power Utility) is proposing a $100 \mathrm{MW}$ solar plant in Upington, Northern province, South Africa. About 14 solar farms have received approval from the National Department of Environmental Affairs of South Africa to construct solar farms ranging from photovoltaics, concentrated photovoltaics to concentrated solar thermal power from the Northern Cape to the Eastern Cape all under IPP (Independent Power Producer) [2]. The biggest ones are Group five $650 \mathrm{MW}$ farms at Kalahari Reserve, Ilangalethu $125 \mathrm{MW}$ farm at Karoshoek and $310 \mathrm{MW}$ at Pofadda.

\section{Working Principles of Power Tower CSP}

The main components of a CSP solar farm are: the solar field/heliostat, receiver, heat transfer fluid/heat exchanger, thermal energy storage thermal cycle/steam turbine/generator and condenser. The simplified diagram of the power tower CSP plant is shown in Fig. 1.

\subsection{The Solar Field}

The solar field is made up of the collectors which consist of mirrors/lenses and the tracking devices known as heliostats [3]. A heliostat is a device that

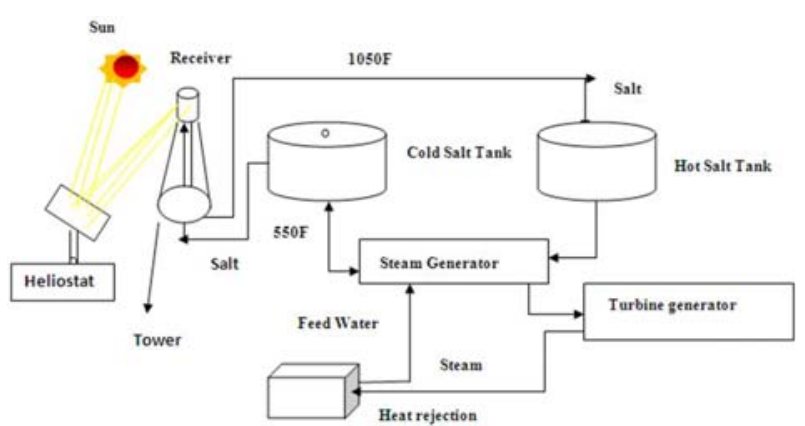

Fig. 1 A simplified diagram of a CSP plant.

includes a mirror which turns so as to keep reflecting sunlight toward a predetermined target compensating the sun's movement. Heliostats are motorized mirrored devices that move to compensate for the changing angle of the sun as it moves across the sky, reflecting its rays onto a fixed target such as a central receiver. In solar tower system, the heliostats are the most important and costly elements of the solar tower constituting $50 \%$ of the total cost of the plant. Therefore, cost saving here would contribute immensely to reducing the unit cost of energy produced by this plant. There are five designs of heliostats.

The following are five types of heliostats: (1) pedestal mounted - common heliostat as shown in Fig. 2; (2) bubble - enclosed membrane-suitable for optical splitting; (3) ganged - all units ganged together with strips of rods; (4) caroused - a large single mirror instead of small $\left(100 \mathrm{~m}^{2}\right)$ units; (5) rotating field heliostats. This field heliostat is the most advanced heliostat with accurate tracking systems.

Extensive evaluation shows that pedestal mounted (100 $\mathrm{m}^{2}$ sizes) heliostat has a cost advantage over other heliostats. The cost is about US $\$ 126$ if 50,000 units are produced per year. Azimuth drive pedestal account for $30 \%$ of the total cost [4].

One way to boost the efficiency of the solar field is by installing spectral splitting filters. This can also be achieved by either of the following methods. The first method is by placing the spectral splitting filters at the heliostats. The second method is the beam down optics.

First, the splitting of the solar spectrum at the heliostats could be achieved by placing narrow 


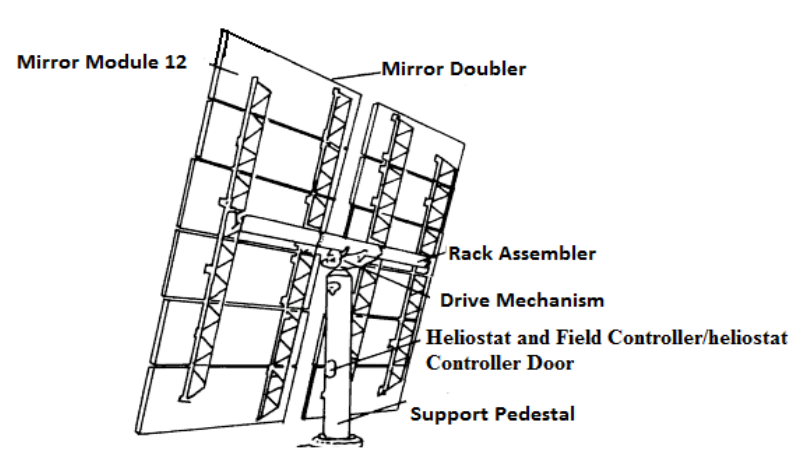

Fig. 2 Pedestal mounted heliostat.

bandpassfilters to isolate waves corresponding to the bandwidth of silicon which is between $600 \mathrm{~nm}$ and 900 $\mathrm{nm}$. An example of a narrow band pass filter is stretched micro structured Mylar membranes over the mirrors described in Ref. [5]. The silicon solar cells can convert a narrow band (600-900 nm) wave with over $60 \%$ efficiency. About 6,000 cells, heat sinks and optical filters would be needed.

Another method is the beam down optics in which the hyperboloid-shaped tower replaces an ordinary receiver. The hyperboloid-shaped tower reflector is used as the spectrum splitter. Its mirror can be made of transparent fused silica glass, coated with a dielectric layer, functioning as a band pass filter. The high energy waves over $1.4 \mathrm{eV}$ could be filtered out and routed to ultraviolent optimized solar cell [6]. The lower reflector mirror is made of silvered borosilicate as shown in Fig. 3.

The rest of the spectrum is passed through a fibre optic cable for further concentration instead of a HTF (heat transfer fluid). Compound parabolic concentrator is used to increase the concentration up to 10,000 suns producing a temperature of about $2,000{ }^{\circ} \mathrm{C}$. This system is popularly known as concentrated photovoltaic and thermal hybrid system $[7,8]$.

\subsection{Black Body Receiver}

An ideal blackbody is a physical body that absorbs all incident electromagnetic radiation. Because of this perfect absorptivity at all wavelengths, a blackbody is also the best possible emitter of thermal radiation which it radiates incandescently in a characteristic

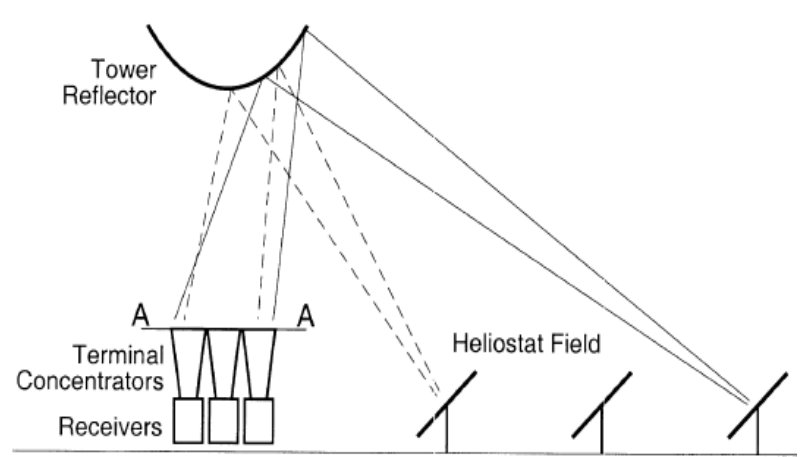

Fig. 3 Reflector beam optics.

continuous spectrum that depends on the body's temperature. Graphite and lampblack are close to blackbodies with emissivity greater than 0.95 . Other blackbodies include cylindrical blackbody solar receiver and black fluid with antireflective device [9]. Here, the receiver consists of an annular cylindrical tube with an aperture parallel to the axis of the cylinder. To reduce the radiative losses, the tube is surrounded by a layer of a thermal insulator [10]. There are two types of receiver namely: external and cavity receiver.

The external receivers are designed to absorb rays coming from all directions. They have very simple designs. The size is determined by the amount of radiation it is meant to absorb and the amount of heat loss. Common losses are by radiation and convention. They are shaped either tubular or spherically.

The cavity receivers are used in most of the commercial large scale solar plants. They have an aperture through which internal reflection ensures that the majority of the radiation that has entered the cavity is absorbed on the internal surface. Heat losses are minimal but more expensive. Once, the heat is absorbed in the cavity, it is transferred to the working fluid [11]. The cavity blackbody can convert incident radiation to heat at $2,000{ }^{\circ} \mathrm{C}$ with an efficiency of $90 \%$.

\subsection{Heat Exchanger/Storage/Transport Facilities}

What makes the CSP electricity unique and cost effective is the ability of the plant to store and despatch energy at times of high demand. A number of energy storage techniques have been studied and these include 
mechanical energy systems which are pumped hydro storage, compressed air storage, flywheels; chemical energy storage such as electrochemical batteries and organic molecular storage. Others are biological energy; SMES (superconducting magnetic energy). So far thermal energy storage has been shown to be the cheapest and one of the most efficient (in the excess of $90 \%$ ). A cost of US $\$ 163 / \mathrm{kWh}$ thermal energy storage was derived by the NREL (National Renewable Energy Laboratory). Once the heat has been realized from the central receiver, it has to be transmitted from the tower to the heat bank which is located at the ground, hence heat transfer fluid is used. Whether the heat exchanger is needed or not depends on the design and the type of materials used.

High temperature storage concept could be studied in two parts; the type of storage which are active or passive storage, the materials used as the storage medium which could be solid, liquid or gases of substances like salt.

Active storage systems are mainly characterized by forced convection heat transfer into the storage material. The storage medium itself circulates through a heat exchanger (this heat exchanger can also be a solar receiver or a steam generator). This system uses one or two tanks as storage media. Active systems are further divided into direct and indirect systems. In a direct system, the heat transfer fluid serves also as the storage medium, while in an indirect system, a second medium is used for storing the heat.

Passive storage systems are generally dual medium storage systems: the HTF passes through the storage only for charging and discharging a solid material. The HTF carries energy received from the energy source to the storage medium during charging and receives energy from the storage when discharging (these systems are also called regenerators). The main disadvantage of the regenerators is that the salt temperature decreases during discharging as the storage material cools down. The second disadvantage is that the heat transfer is low since there is no contact between the HTF and the storage material. Other designs include two-tank direct, two-tank indirect and single tank thermocline.

Single tank thermocline is a form of active indirect storage system where the hot and the cold fluids are stored in the same tank. A thermocline also known as a metalimnion is a thin but distinct layer in a large body of fluid in which temperature changes more rapidly with depth than it does in the above or below it. This system provides one possibility of further reducing the cost of a direct two-tank storage system. Here, the hot and cold fluids are separated because of the stratification and the zone between the hot and the cold fluids is called the thermocline. A filler material is used to help the thermocline effect. Sandia National Laboratories identified the filler material as quartzite rock and silica sands [12].

\subsection{Thermal Storage Materials}

Thermal storage materials are classified in three main categories namely: sensible heat materials, phase change materials and chemical heat storage materials.

Sensible heat storage materials are defined as a group of materials which undergo no change in phase over the temperature range encountered in the storage process. The amount of thermal energy $Q$, stored in joules is given by:

$$
Q=\rho \cdot \hat{\mathrm{c}}_{p} \cdot V \Delta T
$$

where, $\rho$ is the density of the storage material in $\mathrm{kg} / \mathrm{L}$, $\hat{c}_{p}$ is the specific capacity over the temperature range of operation in $\mathrm{J} / \mathrm{kg} \cdot \mathrm{K}, V$ is the volume of the storage material used in $\mathrm{L}$, and $\Delta T$ is temperature range of the operation in ${ }^{\circ} \mathrm{C}$.

The following physical properties are considered for a material to be useful as a sensible heat material; thermal conductivity, inexpensive, thermal diffusivity and low melting point. Sensitive heat store could either be liquid or solid. Liquids are less common because at higher temperature they evaporate hence pressure containers add to the cost of the system. Examples of sensitive heat materials include ceramics, reinforced 
concrete, cast iron, magnesia fire bricks and molten salt. Some CSP where the HTF and the storage material are the same typically molten salt, a mixture of two or more salts are used whose melting point is lower than the melting point of the individual salts. This mixture is known as eutectic mixture; $60 \% \mathrm{NaNO}_{3}$ and $40 \%$ $\mathrm{KNO}_{3}[13]$.

PCM (phase change materials) also known as latent heat storage media refer to those materials that store thermal energy isothermally as latent heat of fusion or evaporation as they change phase from solid to liquid or from liquid to gas. Examples of PCM are paraffin (RT110), HITEC salt $\left(\mathrm{KNO}_{3}-\mathrm{NaNO}_{3}-\mathrm{NaNO}_{3}\right)$, Hydroxides, $\mathrm{CaCO}_{3}, \mathrm{Fe}_{2} \mathrm{CO}_{3}$ [14]. Organic materials are preferred because their operating temperatures which are close to the room temperature.

An advantage of PCM is higher energy density hence lower cost. The disadvantages of PCM are higher complexity of the system, uncertainty of life time cycle and poor conductivity.

Chemical heat storage material is the technology of utilizing the heat of chemical reaction to store thermal energy with the possibility of realizing higher energy process than any other thermal energy storage technologies. Examples of chemical heat storage materials are shown in Table 1.

The research and development in chemical heat storage is focused on heat transformer, CHP (chemical heat pump) in the following aspects: Reaction-phase change, reaction-reaction and reaction separation. Heat transformers are absorption pumps that boost the temperature of industrial waste heat. Currently, the limit of this heat transformation is about $100{ }^{\circ} \mathrm{C}$. Solutions of $\mathrm{LiBr}$ (lithium bromide), $\mathrm{NaOH}$ (sodium hydroxide-water) are commonly used. Advantages of chemical heat storage are highest energy density of three types, suitable for long term storage, wide and flexible operating temperature. The PCM and CHS are still under basic research and are not yet used on any CSP farm. A novel hybrid storage material made of the PCM-Sensible-PCM is developed in Ref. [14]. The
Table 1 Chemical heat storage materials.

\begin{tabular}{|c|c|c|c|}
\hline Reactants & Products & Energy/density & Temperature \\
\hline $\mathrm{NH}_{3}+$ Heat & $\mathrm{N}_{2}+\mathrm{H}_{2}$ & $67 \mathrm{~kJ} / \mathrm{mol}$ & $400-500^{\circ} \mathrm{C}$ \\
\hline $\mathrm{MgO}+\mathrm{H}_{2} \mathrm{O}$ & $\mathrm{H}_{2} \mathrm{O}+$ Heat & $3.3 \mathrm{GJ} / \mathrm{m}^{3}$ & $250-400^{\circ} \mathrm{C}$ \\
\hline
\end{tabular}

advantages of hybrid thermal storage are increased storage capacity, better use of PCM storage capacities, reduction in the cost of storage and improved storage ratio [15].

\subsection{Power Conversion Unit}

The power conversion unit is basically made of the thermodynamic cycle(s), the turbine and a generator. The contents of which vary according to the design of an individual plant.

Most heat engines are based on the Carnot cycle which operates with an efficiency which is dependent on the steam temperature. The Carnot cycle is a theoretical thermodynamic cycle produced by Nicolas Clement and Sadi Carnot in 1824 [16]. It is the most efficient cycle for converting a given amount of thermal energy into work or creating a temperature difference by doing a given amount of work. The Carnot efficiency is given by:

$$
\eta=1-T_{c} / T_{H}
$$

where, $T_{C}$ is the condenser temperature and $T_{H}$ is the input temperature. In practice, other thermodynamic cycles in use are the Rankine cycle and the Brayton cycle.

Rankine cycle is a thermodynamic that converts heat into work. It is sometimes called the practical Carnot cycle. The Rankine cycle is a practical version of the Carnot cycle. The working fluid typically exists as a single phase (liquid or vapour) for the two pressure change processes. There are four processes in a Rankine cycle: (1) the working fluid (water) is pumped from low to high pressure; (2) the high pressure liquid enters a boiler where it is heated at constant pressure by an external heat source to become a saturated vapour; (3) the dry saturated vapour expands through a turbine; (4) the wet vapour then enters a condenser where it is 
condensed at a constant temperature to become liquid.

Other variations of a basic Rankin cycle for better performance are:

In Rankine with reheat, two turbines (high pressure turbine and low pressure turbine) are employed for improving efficiency. Steam after expansion from the high pressure turbine, is sent again to boiler and heated till it reaches superheated condition. It is then left to expand in the low pressure turbine to attain condenser pressure.

In regenerative Rankine cycle, the working sub cooled liquid from the condenser is heated by the steam tapped from the hot portion of the cycle (in-between the turbines) to end up with the saturated liquid. If the steam does not mix with the compressed water from the pump it is called closed feeder heaters.

Organic Rankine cycle uses an organic fluid such as n-pentane or toluene in place of water/steam. This allows the use of lower temperature heat source (70-90 $\left.{ }^{\circ} \mathrm{C}\right)$.

The Brayton cycle also known as Joule cycle is a thermodynamic cycle that describes the working of a gas turbine. It is mostly ran as an open system, external combustion engine as in jet engine however it could also be ran as a closed loop cycle if the exhaust gas is recycled. The basic components of a Brayton cycle are boiler, turbine, condenser and pump as shown in Fig. 4.

A typical Brayton cycle consists of an adiabatic process in which air is drawn for compression, heat is added for combustion (isobaric process), adiabatic expansion of gasses and isobaric rejection in atmospheric pressure. The efficiency of the system could

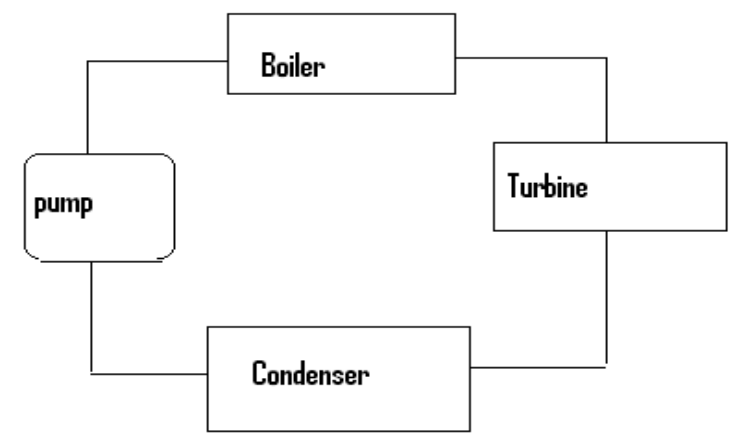

Fig. 4 Brayton cycle. be increased by reheat to increase the temperature, increasing the pressure and by regeneration.

MHD (magneto hydrodynamic) generator is a dynamo that converts thermal/kinetic energy directly into electricity. MHD produces electricity by passing a magnetic field across a hot (about 2,000 ${ }^{\circ} \mathrm{C}$ ) fluid such as an inert gas (helium or caesium seed) which usually in plasma forms. MHD is a very reliable device because there is no moving part. The start-up time is short. It could be used as a topping generator in a combined cycle plant because the output is a flame. The efficiency is over $50 \%$ and it is suitable for a large scale power production [17].

Thermoelectric generators also known as thermo generators are devices which convert heat (temperature differences) directly into electrical energy, using a phenomenon known as the Seaback effect. Classes of materials that have been used for thermo electrics are ordinary alloys of metals (like $\mathrm{Bi}_{2} \mathrm{Te}_{3}$ ), complex crystals (semiconductors like GaAs) and multiphase nanocomposites such as silicon nanowires. The thermoelectrics have not been used as generator because of their high internal resistance which makes them ideal for temperature measuring instruments. Recently, scientists are considering the use of thermoelectrics as generators because they are less complex and have no moving parts. Currently, their efficiency is about $15 \%$ [18]. Micropelt is a manufacturer and a vendor of novel thin film thermoelectric components [19].

The NECs (Nantenna Electromagnetic Collectors) are solar converters that harvest solar energy based on the wave-particle duality nature of the light. Sunlight had been seen as photons which carry pockets of energies and photovoltaic cells have been optimized to capture various energy levels of photons except that these photovoltaic cells have been characterized by low efficiency because of the very wide band of the solar radiation. The nantennas are nano particle antennas which are equipped with nano or molecular diodes. They are often tuned to the frequency of light hence they also known as the optical antennas. They capture 
the solar radiation and convert it to DC signal via the diodes. These antennas have been tested with the infrared waves and have been shown to absorb heat waves (or infrared) with $60 \%$ efficiency [20]. It is proposed that future power plant could reduce cost, durability and increase efficiency by replacing the power conversion unit with the NEC [20]. Within the solar energy spectrum, the infrared (heat) waves possess the longest wavelength hence they are most easily harvested with nano diodes. The NECs currently are targeted for thermal to electricity conversion and if they are successfully developed commercially, they would be ideal for thermal conversion unit of the CSP.

It has been shown that ISCCS (integrated solar combined-cycle system) configuration could reduce the cost of solar power by as much as $22 \%$ [21]. Hybridizing with fossil fuel can be done in various ways: using an auxiliary system to heat the HTF during low insolation; introducing the fossil back up in the steam cycle - the evaporation, superheat or reheat zones. The advantages of the ISCCS are: the solar to electricity conversion; incremental costs for a larger steam turbine compared to the overall unit cost in solar plant only. A solar triple cycle power which is composed of three conversion steps, a topping MHD cycle, an intermediate Brayton cycle and a bottoming steam Rankine cycle was proposed in Refs. [21, 22]. Using a simple model, the efficiency of the entire plant for a range of temperatures could be optimized and the performance of a two level solar combined cycle and a new triple cycle could be compared.

\subsection{Power Cycle Integration Concepts}

The heat generated in the solar absorbers is subsequently used for electric power generation. Different concepts for integration of the solar collector into conventional power cycles are presented and the difference between these concepts is the collector integration within the thermodynamic cycle and the degree of hybridization with fossil power generation (solar share). The following are available.
Option 1: Solar live steam in hybrid Rankine plants as is used in SEGS plants in California [23]. Coupling a solar field with a conventional coal-fired power plant can lead to a lot of savings in $\mathrm{CO}_{2}$ production whenever solar steam is available.

Option 2: Solar gas turbine combined cycle option has possibility of high efficiencies in the medium term cost saving in field investment.

Option 3: Combined heat and power solar plant, this is similar to the combined heat and power, in this option a solar thermal electricity application such as sea water desalination or air conditioner co-firing is possible. Due to a double use solar heat and power, an efficiency of $80 \%$ is possible. Any type of thermal plant could be designed to capture the waste heat.

Option 4: Investigates ISCC based on the high temperature gas turbine and a bottoming steam turbine. The size of the steam turbine in ISCCS is larger than it would be in a conventional combined cycle. Advantages of ISCC are higher solar shares in solar feed water preheating, no solar energy losses due to daily plant start-up and shut-down and the incremental costs for a larger steam turbine are less than the overall unit cost in a solar-only plant.

Option 5: Solar feed water preheating; the solar thermal heat is used for preheating feed-water in large scale conventional Rankine plants, substituting steam that would otherwise be bled from the turbine. Additional electricity can be generated, or fossil fuel be saved depending on the operation mode. This concept is well suited for market introduction of new collector types since it reduces the risk of lowering the investment costs that would otherwise be needed for a new steam cycle.

Option 6: Solar process heat application such as space cooling of buildings and refrigeration are ways to dynamically cut the initial cost of investment in CSP.

\section{Simulation of Molten Salt Power Tower CSP}

In order to determine the economic viability of the molten salt power tower CSP in South Africa, a 
summary of the economic model run using SAM is presented in this section.

LCOE (levelized cost of energy) is broadly used in the industry to determine the electricity tariff system and it is given by the formula:

$$
L C O E=\frac{\sum_{n=0}^{N} \frac{C_{n}}{(1+d)^{n}}}{\sum_{n=1}^{N} \frac{Q n}{(1+d)^{n}}}
$$

where:

$Q_{n}(\mathrm{kWh})$ is electricity generated by the system in year;

$N$ is the analysis period in years;

$R_{n}$ is the project revenue from electricity sales in year $n$;

$C_{0}$ is the project's initial cost;

$d$ is the discount rate.

The solar multiple of 3.2 and thermal energy storage in hours of $15 \mathrm{~h} \mathrm{LCOE}$ of R1.05/kWh was realized over the analysis period of $20 \mathrm{yrs}$.

\section{Conclusions}

Concentrated solar power is a proven technology. Test plants have been setup and are running in the United States of America, Spain and other parts of the world. South Africa is about to begin a number of CSP projects. The main advantage of CSP is the ability to store and dispatch electricity at peak demand periods. Apart from electricity, the CSP can also produce industrial heat even at greater efficiency than it produces electricity. The main challenge facing CSP development is that of low efficiency in producing electricity hence research efforts are directed towards finding better materials as discussed in this paper. Another research that has not been covered is the use of optimization techniques to enhance the efficiency of the CSP plant. However, at the current state of research in CSP technology, the simulation results show that the cost of CSP electricity is viable in the South Africa tariff system.

\section{Acknowledgments}

This work is based on research supported wholly by the National Research Foundation of South Africa UID: 71967; UID: 83977.

\section{References}

[1] A.F. Garcia, E. Zarza, L. Venezuela, Renewable and sustainable energy review, Science Direct 14 (7) (2010) 1695-1721.

[2] National Department of Environmental Affairs, Savannah Environment (Pty) Ltd. [Online], www.savannahsa.com.

[3] C. Wyman, J. Castle, F. Kreath, A review of collector and energy storage technology for intermediate temperature application, Solar Energy 24 (6) (1980) 517-540.

[4] G.J. Kolb, S.A. Jones, M.W. Donnelly, D. Gorman, R. Thomas, R. Davenport, Heliostat Cost Reduction Study, Sandia report, SAND 2007-3292, Unlimited Release, June 2007.

[5] V. Hejmandi, M.M. Shin, B. Kress, A. Gilberto, Novel solar co-generation trough system based on stretched micro structure Mylar film, SPIE Eco-Photonics 8065 (2011) 1-10.

[6] A. Segal, M. Epstein, A. Yogev, Hybrid concentrated photovoltaic and thermal power conversion at different spectral bands, Solar Energy 76 (5) (2004) 591-601.

[7] W. Chen, H.A. Rahman, S.P. Rao, Design of a FFSC (fluorescent fiber solar concentrator) and outdoor testing for remote indoor day lighting, in: 15th International Conference on Responsive Manufacturing (ICRM), Ningbo, China, Jan. 2010, pp. 320-329.

[8] R.D.M.C. Connell, J. Thompson, A hybrid solar concentrator for electrolysis and production of Hydrogen, www1.eere.energy.gov.

[9] D.A. Boyd, R. Gejewski, R. Shift, Cylindrical blackbody solar energy receiver, Solar Energy 18 (5) (1976) 395-401.

[10] C. Suter, P. Tomes, A. Weidenkaff, A. Steinfeid, A solar cavity-receiver packed with an array of thermoelectric converter modules, Solar Energy 85 (7) (2011) 1511-1518.

[11] C.C. Newton, A concentrated solar thermal energy system, Master Thesis, Famufsu College of Engineering, The Florida State University, 2007.

[12] J.E. Pacheco, S.K. Showalter, W.J. Kolb, Development of a molten-salt thermocline thermal storage system for parabolic trough plants, American Society of Mechanical Engineers Journal of Solar Energy Engineering 124 (2) (2002) 153-159.

[13] D. Kearney, U. Herrmann, P. Nava, B. Kelly, R. Mahoney, J. Pacheco, et al., Assessment of a molten salt heat transfer fluid in a parabolic trough solar field, Journal of Solar Energy Engineering 125 (2) (2003) 170-176. 
[14] A. Gil, M. Medrano, I. Martorell, A. Lazaro, P. Dolado, B. Zalba, et al., State of the art on high temperature thermal energy storage for power generation: Part 1 -Concepts, materials and modellization, Renewable and Sustainable Energy Reviews 14 (1) (2010) 31-55.

[15] A. Gil, P. Arce, I. Martorell, M. Medrano, L.F. Cabeza, State of the Art of High Temperature Storage in Thermosolar Plants, Efstock, 2009.

[16] R. Fox, Watt's expansive principle in the work of Sadi Carnot and Nicolas Clement, Notes and Records of the Royal Society of London 24 (2) (1970) 233-253.

[17] J. Grul, Coal-fired Open Cycle Magneto-Hydrodynamic Power Plant Emissions and Energy Efficiencies, MIT Energy Lab. report, \#MIT-EL 78-018, MIT, 1977.

[18] H. Bottner, Micropelt miniaturized thermoelectric devices: Small size, high cooling power densities, short response time, in: 24th International Conference on
Thermoelectrics, South Carolina, USA, 2005.

[19] A.I. Hochbaum, R. Chen, R.D. Delgado, W. Liang, E.C. Garnett, M. Najarian, et al., Enhanced thermoelectric performance of rough silicon nanowires, Nature 451 (7175) (2008) 163-167.

[20] J. Sol, Theory and manufacturing processes of solar nano-antenna electromagnetic collectors, Energy Engineering 132 (1) (2010) 11-14.

[21] A. Kribus, A high-efficiency triple cycle for solar power generation, Solar Energy 72 (1) (2002) 1-11.

[22] J. Servert, G.S. Miguel, D. Lopez, Hybrid solar-biomass plants for power generation, technical and economic assessment, Global Nest Journal 13 (3) (2011) 266-276.

[23] Assessment of the World Bank Group, GEF Strategy for the Market Development of Concentrating Solar Thermal Power, the International Bank for Reconstruction and Development, World Bank, Vol. 1, 2006. 